\title{
Téoros
}

Revue de recherche en tourisme

\section{Quels sont les obstacles au développement du marché québécois des « beds and breakfasts " ?}

\section{Vincent Sabourin}

Volume 12, numéro 3, octobre 1993

URI : https://id.erudit.org/iderudit/1077939ar

DOI : https://doi.org/10.7202/1077939ar

Aller au sommaire du numéro

Éditeur(s)

Université du Québec à Montréal

ISSN

0712-8657 (imprimé)

1923-2705 (numérique)

Découvrir la revue

Citer cet article

Sabourin, V. (1993). Quels sont les obstacles au développement du marché québécois des « beds and breakfasts » ? Téoros, 12(3), 43-46.

https://doi.org/10.7202/1077939ar d'utilisation que vous pouvez consulter en ligne. 


\section{Quels sont les obstacles au développement du marché québécois des «beds and breakfasts»? \\ Vincent Sabourin}

Depuis les cinq dernières années, le marché nord-américain des *beds and breakfasts* a connu une forte croissance et s'est considérablement structuré. D'une part, la demande pour ce type de services touristiques s'est accrue. D'autre part, la concurrence entre les différents réseaux des différentes villes nord-américaines est devenue plus intense.

Dans ce contexte, les *beds and breakfasts du Québec, organisés sous l'égide de la Fédération des Agricotours, se sont efforcés depuis les derniêres années de bénéficier de la croissance du marché afin de développer leur réseau. Les pressions gouvernementales afin d'accroître l'autofinancement du réseau ont amené la Fédération des Agricotours à évaluer et développer de nouvelles stratégies de développement exprimées dans le cadre plus large du plan d'orientation d'Agricotours élaboré en 1984:

\section{Cette tendance afférente au développement des Gites du passant devrait conduire à l'adoption par la féderation d'une mission et de buts généraux axér sur le développement et le soutien du atourisme en résidences privéesw, concept englobant de fagon phus conforme la réalité actuelle. Dansle même sens, la fédíration devra envisager de se donner un nouveau nom corporatif, dès que la pénétration du milieu urbain le justifiera, afin davoir une identification qui à la fois inclus et excède l'agrotourisme ou le tourisme en milieu rural (p. 73).}

L'objectif général de cette recherche fut d'identifier les obstacles économiques et commerciaux au développement du marché des gîtes connus sous le nom de abeds and breakfasts» et dans une perspective à

Monsieur Vincent Sabourin est professeur au departement des sciences administratives de l"Université du Québec a Montróal. L'auteur tient à remercier madame Odette Chaput, directrice generale de la Feddration des Agricotours durant tout notre projet de recherche. long terme, les défis s'y rattachant pour la Fédération des Agricotours qui est le réseau regroupant la plus importante proportion des gites au Québec.

\section{La méthodologie}

La méthodologie a comporté un certain nombre d'étapes.

Premièrement, une recherche d'information évalua systématiquement la situation des gites québécois en comparaison avec des régions similaires. Une cueillette d'information et des entrevues furent complétées auprès des réseaux des abeds and breakfastss aux Etats-Unis: Chicago beds and Breakfasts, beds and Breakfasts Connection (Philadelphie), Urban Ventures New York, Twins Fork B \& B (Valley Forge), B \& B Leagues (Washington), Greater Boston Hospitality, B \& B Philadelphie; en Ontario et les Maritimes: Nova Scotia Farm \& Country Vacations B \& B Homes, New-Brunswick B \& B Association, Ontario B \& B Association.

Deuxièmement, la réalisation de deux tables de discussion et entrexues avec des consommateurs des services de gites ${ }^{(1)}$ permirent d'évaluer plusieurs aspects des gites: le rôle des sources d'information dans la décision d'achat des services du réseau, le moment et le lieu de l'achat, le type d'utilisation.

\section{La position des gites dans le marché touristique}

Dans l'ensemble des villes nord-américaines, les informations recueillies ont mis en lumière que les réseaux de *beds and breakfasts sont dans une phase de structuration. La demande pour ce type de service touristique a connu une croissance au cours des cinq dernières années. Les gittes attirent de plus en plus une clientèle qui leur sont fidèles et apportent des sources de revenus de plus en plus stables à cette industrie. Les réseaux de gîtes des villes nord-américaines ont bénéficié d'une aug- mentation de leur taille au cours des dernières années. Certains réseaux regroupent plusieurs centaines de gites. Ainsi, le réseau Urban Ventures est un des réseaux opérant dans la ville de New York et regroupe plus de 700 gites. La majorité des villes américaines fonctionnent avec un nombre de plus en plus limité de réseaux soient entre 3 et 12 organismes et rassemblent une majorité de gites. La proportion d'indépendants (c'est-à-dire de gîtes non affiliés à un réseau) tend à diminuer au cours des années.

À partir de ces données, nous pouvons estimer que le développement du marché des «beds and breakfasts $»$ au Canada et au Québee possède près d'une dizaine d'années de retard sur le marché américain. Pour une majorité de gîtes, ce type de service constitue un revenu d'appoint plutôt qu'une source principale de revenus. Les gîtes fonctionnent au Canada selon un mode plus artisanal qu'aux États-Unis. Ils opèrent nettement plus souvent d'une façon décentralisée. Les réservations sont prises sur une base individuelle et la plupart des gites font leur propre marketing.

Sur le plan du marketing, les réseaux de abeds and breakfasts (surtout aux EtatsUnis) donnent de plus en plus un service dont le professionnalisme s'apparente à celui des réseaux hôteliers. L'industrie du wbeds and breakfasts $\%$ opère avec des normes et des standards de fonctionnement de plus en plus homogènes. On note par exemple que les politiques de réservation, les niveaux de service et les prix sont de plus en plus semblables à travers les réseaux. Ceci s'explique aussi par le fait que le consommateur est expérimenté et connaît mieux la valeur et les exigences de service d'un séjour dans un gîte.

Au Québec, le marché entre dans une phase de structuration et ce, plus spécialement dans la région métropolitaine. Montréal a des réseaux qui regroupent plus de cinquante gites en période estivale. Ces réseaux font de la publicité et occupent une proportion de plus en plus grande de l'es- 
pace publicitaire dans les guides et dépliants touristiques.

D'une façon spécifique, la forte croissance des réseaux de gîtes s'explique par les économiesd'échelled'un regroupement. Faire partie d'un réseau permet de diminuer plus particulièrement deuxtypes decoûts, soient la publicité-promotion et les coûts de réservation:

a) l'adhésion à un réseau donne accès à un levier marketing plus important puisqu'elle permet de partager les coûts de conception, de production et d'achat de l'espace publicitaire et de la promotion;

b) une adhésion à un réseau diminue les coûts de récervation des gittes. Une partie du temps et des énergies mises à la réservation par les gîtes peut être dévolue à l'accueil et au service du consommateur.

Le marché des grandes villes américaines voit apparaître de grandsréseaux centralisés et organisés à l'aide de centrale de réservation. La centrale de réservation permet d'offrir un meilleur service et de doter le siège social d'un budget plus important pour la publicité et la promotion. Des sommes additionnelles proviennent de la commission que prennent les centrales sur les réservations des consommateurs.

Au Canada et au Québec, les réseaux de *beds and breakfasts sont encore structurés d'une façon plus ou moins artisanale. Dans l'ensemble, ill n'existe à peu près pas decentrales deréservation importantes, les gites font leur propre publicité de façon indépendante et disposent à ce stade de leur développement, de peu de moyens financiers pour faire face à la concurrence des grandes chaînes hôtelières. On note par exemple, que le prix d'une chambre est nettement plusélevée auxÉtats-Unisqu'au Canada, ce qui signifie que le consommateur américain est prêt à payer davantage pour un séjour dans un gite que le consommateur canadien. Le tableau 1 illustre les différences de prix.

Toutefois, comme dans la plupart des marchés de consommation, nous pouvons croire que les tendances américaines apparaîtront sous peu au pays. Les réseaux canadiens de *beds and breakfasts's'apparenteront, de plus en plus d'ici 5 à 10 ans, aux réseaux américains. Ils s'organiseront

Tableau 1

L'établissement des prix (prix moyen)*

Simple

Double

50 gites du Massachussets

$53 \$$

$68 \$$

Centrale de réservation aux

Etats-Unis (Urban Ventures, $\mathrm{n}=50$ )

$47 \$$

$68 \$$

Ontario Beds and Breakfasts

Association

$42 S$

$52 \$$

Nouvelle-Écosse

285

365

* En dollars US, données de 1989 ajustées pour 1990 à $5 \%$ d'inflation.

à l'aide d'un marketing plus systématique ayant les caractéristiques suivantes: centrale de réservation, utilisation de carte de crédit, conception de guides touristiques et budgets publicitaires plus importants.

Au Québec, le marché présente de grandes opportunités de développement. La notoriété des gittes se situe aux environs de 20 \% (rapport MLCP, 1987) pour la population québécoise effectuant des voyages. Ceci est faible pour générer des revenus importants permettant des investissements dans la mise en marché.

Dans un tel contexte, il est important que les gittes organisent leurs efforts afin de prendre la place qui lui revient dans le marché d'ici les cinq prochaines années. Le cas échéant, d'autres réseaux d'hébergement se développeront et vont s'accaparer peu à peu une partie de plus en plus importante de ce marché. Cette menace pointe délà à l'horizon avec l'organisation de réseaux centralisés de réservation dans la région métropolitaine et la capitale.

Notre analyse de la situation des gites du Québec et de son principal regroupement, la Fédération des Agricotours, a mis en lumière l'obstacle suivant au développement du marché : les gittes ne bénéficient pas d'un levier marketing et financier suffisants pour financer son développement. La structure de financement et le marketing actuel ne permet pas aux gites de se créer un levier permettant de soutenir la croissance d'un réseau. La figure 1 illustre la situation des gîtes.
Les gites doivent développer un levier marketing leur permettant d'accroître leurs revenus et disposer de ressources supplémentaires pour mieux se faire connaître auprès des consommateurs et structurer leurs services. L'examen de la situation des gites nous mène aux conclusions suivantes. Leprincipal obstadeau développementdu réseau des gîtes est qu'il n'existe pas d'organismes disposant de revenus suffisants pour développer un levier marketing lui permettant de s'imposer comme le réseau des gîtes du Québec. Ceci est essentiellement dû au fait que les gites conservent $100 \%$ des bénéfices supplémentaires des efforts de marketing effectués par des réseaux comme la Fédération des Agricotours.

Plusieurs solutions s'offrent aux gites au Québec, soient la misesur piedd'opération de pré-enregistrement, l'élaboration d'un système de réservation par carte de crédit etl'organisation d'un système centralisé de réservation. Ces solutions pourraient être mis en oeuvre par des réseaux de gîtes.

\section{Une opération de pré-enregistrement}

Une des façons d'augmenter le volume de ventes du réseau est d'effectuer une opération de pré-enregistrement (en anglais «prébooking») durant la période de planification des vacances, soit en avril-mai-juin. Rappelons que ce marché tend à avoir un grand problème de rotation de la clientèle $(1,45$ nuitée par client en 1987) 
Figure 1

La situation des gites

Réseau artisanal

Faibles revenus des gites

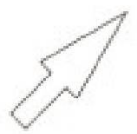

Faibles rentrés de fonds

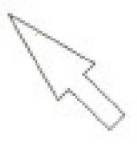

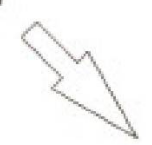

Peu de revenus au siège social des réseaux de gîtes

Pas de levier financier

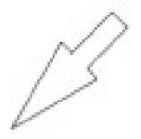
et marketing

ristourne sera suffisamment faible pour ne pas mécontenter et démotiver les propriétaires de gîtes. Elle est sensée être suffisamment importante pour payer les frais d'administration de l'opération et dégagera une marge bénéficiaire. Cette marge bénéficiaire pourrait permettre de constituer un fond de développement pour les réseaux.

\section{Un seul numéro de téléphone}

Afin de contrôler les revenus supplémentaires et l'information sur le réseau, il est nécessaire que les nouveaux dients soient administrés par une centrale de réservation. On présente dans la publicité un seul numéro soit celui de la centrale de réservation des gites.

\section{Une ligne watt 1-800}

Une ligne watt de type 1-800 recevant les appels de consommateurs créera un achalandage de clientèles. Il est important à moyen terme qu'une ligne watt soit disponible pur les régions extérieures: ÉtatsUnis, Ontario, Maritimes.

En somme, le système centralisé de réservation convient bien à un réseau artisanal semi-professionnel mais n'est pas nécessairement approprié à un réseau de plus grande dimension. Notre recherche aupres des réseaux de abeds and breakfasts» à travers les Etats-Unis, en Ontario et dans les Maritimes montre qu'une centrale des réservation peut devenir un outil de marketing et de financement pour les réseaux de gites. D'ici 5 à 10 ans, l'élaboration d'un système centralisé de réservation sera probablement nécessaire pour de nombreux gites et ce, pour les raisons suivantes:

\section{Des rếservations par cartes de crédit}

$\mathrm{La}$ décision de réserver dans un lieu de séjour est, dans une forte proportion, une

\section{L'organisation d'un système centralisé de réservation}

Un système centraliséde réservation pourrait fonctionner selon trois modalités :

Une ristourne sur les ventes supplémentaires générées pour les gîtes

action impulsive. C'est pourquoi il est Le montant du dépôt quant à lui, est de 25 \% de la valeur du séjour et se fait à l'aide de 政 mateurset de faire une meilleure planification de la carte de crédit pourrait être un outil de marketing important pour les membres.

La normenord-américaine pour un système de réservation est de $20 \%-25 \%$. Cette norme est considérée comme un standard de fonctionnement de l'industrie américaine des abeds and breakfasts\%. Cette
Une réservation phus rapide et plus facile. Le consommateur n'a pasà effectuer lui-même le magasinage auprès des différents gîtes. Ce système de réservation permet de faire affaires plus facilement avec le réseau. Les coûts de transaction avec les gites sont sous la responsabilité du réseau.

Une meilleure réponse aux besoins du consommateur. Ce système de réservation répond mieux aux besoins du consommateur. Il permet à l'agent de réservation de donner une information plus objective sur les différrents gîtes et de jouer le rôle de conseiller 
touristique. En comparaison, le système actuel ne permet pas au consommateur de communiquer avec une personne indépendante qui évalue et place sa demande. Les informations sur le marché des gites montrent la nécessité d'informer, «d'éduquer» et de sécuriser les acheteurs lors de l'achatà l'aidedece type de service(MLCP, 1987, p. 145). De plus, ce système répond mieux aux besoins du consommateur parce qu'il permet d'offrir plus facilement un service bilingue.

Diminution de l'importance du réseau comme fonction de dépannage. Ce système diminue la fonction de adepannages que tend trop souvent à jouer le réseau Agricotours et permet de mieux rejoindre des acheteurs faisant une planification de leurs vacances. Les données montrent que la majorité des séjours furent d'une à deux journées et ce, dans un seul gîte (MLCP, 1987, p. 144). Ceci permettra de passer d'une utilisation de dépannage à une utilisation qui soit davantage de type oplanification-réservations.

\section{L'accueil d'une clientèle à plus hauts revenus}

On sait que les voyageurs à plus hauts revenus démarchent moins car ils sont généralementmoins sensibles aux prix. Un système centralisé de rếservation pourrait permettre de mieux servir cette clientèle en diminuant le nombre d'étapes dans le processus d'achat.

\section{Un meilleur contrôle de la qualité des services offerts par les gîtes}

Un des avantages d'un système centralisé de réservation est de permettre un meilleur contrôle de la qualité des services offerts par les gites. Lorsque le consommateur rappelle pour choisir un autre gite, l'agent d'information peut prendre note de son degré de satisfaction ou de son mécontentement de son dernier séjour et peut effectuer les démarches nécessaires afin d'améliorer le service d'un gite. De plus, les consommateurs peuvent communiquer leur insatisfaction directement et sans frais au siège social. Ceci est une information précieuse pour le réseau. Rappelons quele réseau des gîtes est associé à *une image d'hébergement d'appoint, possédant un caractère improvisé et mal organisé»
(MLCP, 1987, p. 145). En somme, le réseau doit se départir de son caractère artisanal en proposant au consommateur un système dont l'organisation est comparable aux autres réseaux hôteliers ${ }^{(2)}$. Un système centralisé de réservation est un pas dans cette direction.

\section{Un monitoring de la demande des consommateurs}

Un système centralisé de réservation permet de faire un monitoring des différentes demandes. L'agent d'information pourta recommander davantage les gites dont le tauxde satisfaction est plus élevéou moduler levolume des réservations en fonction dela disponibilité et des goûts particuliers des proprićtaires de gites. Lors d'entrevues réalisées aux États-Unis et au Canada anglais, plusieurs gites nous ont avoué être passés d'un système décentraliséa un réseau centralisé de réservation en partie parce que les membres en avaient assez de gérer les problèmes de réservation et de marketing et préféraient se concentrer sur leurs principales compétences, soient l'accueil et le service aux voyageurs. Les gites québécois semblent plus fermés que leurs compatriotes anglo-saxons sur cette question.

\section{Un contrôle des ventes et de l'efficacité de la commercialisation}

Un système de réservation centralisé permet de connaître tout au cours de l'année d'une façon fiable, l'évolution des revenus du réseau par région et par gîte. Deplus, les informations compilées lors des réservations (nom, adresse, téléphone, âge, occupation des clients, source d'information, etc.) seront un outil de promotion important pour tracer un portrait de la clientèle, aller chercher de la commandite et choisir les médias pour la publicité. Cette information permet aussi d'ajuster les efforts de marketing tout au cours de l'année. Ce système permet de contrôler spécifiquement l'efficacité de la publicité et des promotions. Une vérification permet à l'agent de réservation de prendre note des promotions les plus efficaces.

Quels sont les inconvénients d'un système centralisé de réservation?

Les coüts supplémentaires. Un système centralisé de réservation représente des coûts supplémentaires qui sont assumés par le siège social alors que dans un système décentralisé, ce sont les gîtes qui assument les coûts (temps et énergie) associés à la réservation. Agricotours a fait les frais d'une telle expérience.

La réristance des gîtes. Un des inconvénients d'un système centralisé de réservation pourrait être la résistance des gites. Les propriétaires de gites se considèrentcomme des entrepreneurs indépendants qui peuvent être méfiants à l'idée de voir le siège social contrôler le trafic des réservations. Il $\mathrm{y}$ aura un travail important à faire auprès des gittesindépendants afin de les convaincre des avantages du nouveau système. Notons pour terminer qu'un programme de commercialisation similaire a permis à un groupe d'hôteliers indépendants de se faire connaître sous le nom de la chaîne hôtelière Hôte et d'accroître significativement leurs ventes auprès de sa clientèle affaires au cours des dernières années.

Notee

(1) Nous utiliserons, dans cet article, le terme agites comme traduction française de abeds and breakfastsw.

(2) Notons quiun systeme bien organise ne signifie pas pour autant que le réseau doive perdre son cachet chaleureux et personnel.

MINISTERE DU LOISIR, DE LA CHASSE ET DE LA PECHE, Etude sur les rbasaux d' habergement. Volume 1: Aubergee de jeunesses, gites du passant et fermes de vacances, avril 1987. 